\title{
Temperature dependence of symmetry energy of finite nuclei
}

\author{
J. N. DÆ and S. K. Samaddar \\ Saha Institute of Nuclear Physics, 1/AF Bidhannagar, Kolkata 700064, India
}

\begin{abstract}
The temperature dependence of the symmetry energy and the symmetry free energy coefficients of atomic nuclei is investigated in a finite temperature Thomas-Fermi framework employing the subtraction procedure. A substantial decrement in the symmetry energy coefficient is obtained for finite systems, contrary to those seen for infinite nuclear matter at normal and somewhat subnormal densities. The effect of the coupling of the surface phonons to the nucleonic motion is also considered; this is found to decrease the symmetry energies somewhat at low temperatures.
\end{abstract}

PACS numbers: 21.10.Dr, 21.30.Fe, 21.65.Ef, 26.30.-k

Keywords: nuclear matter, hot nuclei, Thomas-Fermi approach, symmetry energy

\section{INTRODUCTION}

The nuclear symmetry energy reflects the energy cost in converting the isospin asymmetric nuclear matter to the symmetric one. Conventionally, as part of global mass formulas in the framework of the liquid-drop model (i.e., ignoring pairing and shell-corrections), the symmetry energy $E_{s}$ of an even-even or odd-odd finite nucleus with $N$ neutrons and $Z$ protons is given from the expression $E_{s}=E(N, Z)-E(A / 2, A / 2)=e_{\text {sym }} \frac{(N-Z)^{2}}{A}$ where $A=N+Z, E(N, Z)$ is the energy of the nucleus barring the Coulomb part, and $e_{\text {sym }}$ is the symmetry energy coefficient. For infinite nuclear systems, the value of $e_{s y m}$ is usually taken in the range of $30-34 \mathrm{MeV}[1-3]$. Apart from the evident basic role of symmetry energy in the correct description of the nuclear binding along the periodic table and a broad understanding of the nuclear drip lines, it is of seminal importance in giving a proper description of the dynamical evolution of the core-collapse of a massive star and the associated explosive nucleosynthesis. A large (small) magnitude of $e_{\text {sym }}$ inhibits (accelerates) change of protons to neutrons through electron capture [4, 5]. This subtly changes the equation of state (EOS) of the dense stellar matter and influences the dynamics of the collapse and explosive phase of a massive star.

Stellar matter in the collapse or bounce phase is warm, knowledge about the temperature dependence of the symmetry energy is therefore a concern of paramount importance. It is also an important ingredient in properly accounting for the multiplicity distributions of the fragment masses and charges and also their isotopic distributions in multifragmentation of hot nuclear systems. Temperature dependence of nuclear symmetry energy and the symmetry free energy $F_{s}$ (defined likewise $E_{s}$ as $F_{s}=F(N, Z)-F(A / 2, A / 2)=f_{\text {sym }} \frac{(N-Z)^{2}}{A}$, where $F$ is the free energy) for homogeneous infinite nuclear matter

*Electronic address: jn.de@saha.ac.in has thence been studied; model calculations show that $E_{s}$ for infinite matter decreases with temperature while $F_{s}$ exhibits the opposite temperature dependence [6]. The temperature dependence becomes more prominent at low densities, particularly for $F_{s}$. In stellar core-collapse or bounce phase, matter is, however, not homogeneous, it is nucleated [7, 8] and therefore it is important to know how the symmetry energies of finite nuclei behave with increasing temperature. The present communication is aimed to understand this feature.

Calculations on symmetry energy for finite nuclei in a low temperature domain $(T \leq 2 \mathrm{MeV})$ have been done earlier by Donati et.al [9] for applications in core-collapse supernova simulations. At finite temperature, the symmetry coefficient was found to be somewhat larger than for cold nuclei $(T=0)$ hindering electron captures. The calculations were schematic, but takes into account coupling of the nucleons to the dynamical surface phonons that results in an increased effective nucleon mass, the so called $\omega$-mass $m_{\omega}$ embodying nonlocality in time [10]. This effective mass $\left(m_{\omega}\right)$ decreases with temperature, resulting in an increase in the symmetry energy. Calculations by Dean et.al [11] in a shell model Monte Carlo (SMMC) framework support these findings. These calculations have also been done in the same limited temperature range and may suffer from shortcomings in the isospin dependence of the schematic residual interaction they have used. The symmetry coefficients calculated by them are much below the accepted nominal values.

The present calculations have been done in a broader temperature domain $(T \leq 8 \mathrm{MeV})$ to have a better understanding of the temperature dependence of symmetry energies in atomic nuclei. Finite temperature ThomasFermi framework with the subtraction technique [12] has been employed for this purpose. Two effective interactions, namely the modified Seyler-Blanchard (SBM) [13, 14] and the $\mathrm{SkM}^{*}$ [15] interactions that give practically the same symmetry coefficient $(\sim 31 \mathrm{MeV})$ at their respective saturation densities have been used in our calculations. Dynamical changes in the energy mass $m_{\omega}$ with temperature is also taken into account following the prescription of Refs. [16, 17]. 
ical framework is briefly outlined. In Sec. III, results and discussions are given. The concluding remarks are presented in Sec. IV.

\section{THEORETICAL FRAMEWORK}

The methodology employed to calculate the symmetry energy and symmetry free energy of finite nuclei as a function of temperature is outlined in the following.

\section{A. Effective interactions}

In describing the hot nucleus in the finite temperature Thomas-Fermi (FTTF) approach, we have chosen, as already stated, two effective interactions with nearly the same symmetry coefficient $\left(e_{\text {sym }} \sim 31 \mathrm{MeV}\right.$ at the respective saturation density for nuclear matter). Both these interactions are density dependent and both have a quadratic momentum dependence. The $\mathrm{SkM}^{*}$ interaction has common usage and FTTF calculations with this interaction [12, 15, 18] have been performed before, with much success. The Seyler-Blanchard [19] interaction has also been very successfully used by Myers and Swiatecki [20, 21] in the context of the nuclear mass formula. The modified version of this interaction (SBM) is found to reproduce the ground state binding energies, charge rms radii, Giant Monopole Resonance (GMR) energies etc., very well for a host of nuclei from ${ }^{16} \mathrm{O}$ to very heavy systems [14, 22]. The properties of pure neutron matter calculated with this type of interaction is also seen to be in good agreement [23] with those obtained from other sophisticated interactions.

The SBM interaction is given by,

$$
\begin{array}{r}
v_{e f f}(r, p, \rho)=C_{l, u}\left[v_{1}(r, p)+v_{2}(r, \rho)\right], \\
v_{1}=-\left(1-p^{2} / b^{2}\right) f\left(\mathbf{r}_{1}, \mathbf{r}_{2}\right), \\
v_{2}=d^{2}\left[\rho\left(r_{1}\right)+\rho\left(r_{2}\right)\right]^{\kappa} f\left(\mathbf{r}_{1}, \mathbf{r}_{2}\right),
\end{array}
$$

with

$$
f\left(\mathbf{r}_{1}, \mathbf{r}_{2}\right)=\frac{e^{-\left|\mathbf{r}_{1}-\mathbf{r}_{2}\right| / a}}{\left|\mathbf{r}_{1}-\mathbf{r}_{2}\right| / a}
$$

The isospin dependence in the interaction is brought through the different strength parameters $C_{l}$ for likepairs (n-n,p-p) and $C_{u}$ for unlike pairs (n-p). The relative separation of the nucleons in configuration and momentum space are given by $r=\left|\mathbf{r}_{1}-\mathbf{r}_{\mathbf{2}}\right|$ and $p=\left|\mathbf{p}_{\mathbf{1}}-\mathbf{p}_{\mathbf{2}}\right|$. The densities at the sites of the two interacting nucleons are given by $\rho\left(\mathbf{r}_{1}\right)$ and $\rho\left(\mathbf{r}_{\mathbf{2}}\right)$. The range of the interaction is $a, b$ and $d$ are the measures of the momentum and density dependence in the interaction and $\kappa$ controls the stiffness of the nuclear EOS.

The five parameters $C_{l}, C_{u}, a, b$, and $d$ for a fixed value
TABLE I: The parameters of the SBM effective interaction (in $\mathrm{MeV}$ fm units)

\begin{tabular}{cccccc}
\hline \hline$C_{l}$ & $C_{u}$ & $a$ & $b$ & $d$ & $\kappa$ \\
\hline 348.5 & 829.7 & 0.6251 & 927.5 & 0.879 & $1 / 6$ \\
\hline \hline
\end{tabular}

ing (i) the volume energy coefficient for symmetric nuclear matter $\left(a_{v}=-16.1 \mathrm{MeV}\right)$, (ii) saturation density of normal nuclear matter (taken as $\rho_{0}=0.1533 \mathrm{fm}^{-3}$, corresponding to the radius parameter $r_{0}=1.16 \mathrm{fm}$ ), (iii) the volume symmetry coefficient $e_{\text {sym }}=31 \mathrm{MeV}$, (iv) the surface energy coefficient of symmetric nuclear matter $\left(a_{s}=18.01 \mathrm{MeV}\right)$, and $(\mathrm{v})$ the energy dependence of the real part $V_{A n}$ of the nucleon-nucleus optical potential $\left(\frac{d V_{A n}}{d E}=0.30\right)$.

The procedure for determining the parameters are given in detail in Ref. [13]. The parameter $\kappa$ is obtained by reproducing the giant monopole resonance energies of a large number of nuclei employing the scaling model [22]. The parameters of the interaction are listed in Table. I. These parameters differ somewhat from the ones used in Ref. [14] where the volume symmetry coefficient $e_{\text {sym }}$ was taken as $34 \mathrm{MeV}$. In the present communication, it is taken as $31 \mathrm{MeV}$, a value commonly used by several authors with some justification from experimental findings [3]. The effective $k$-mass of the nucleon $m_{k}$ coming from the momentum dependence of the effective interaction, comes out to be $0.62 m$ for symmetric matter, where $m$ is the nucleon mass. The isoscalar volume incompressibility $K_{\infty}$, symmetry incompressibility $K_{\text {sym }}\left[=\left(9 \rho_{0}^{2} \frac{\partial^{2} e_{s y m}}{\partial \rho^{2}}\right)_{0}\right]$ and the symmetry pressure $L\left[=\left(3 \rho_{0} \frac{\partial e_{s y m}}{\partial \rho}\right)_{\rho_{0}}\right]$ are 240 , -101 and $59.8 \mathrm{MeV}$, respectively for the SBM interaction. The corresponding values for $\mathrm{SkM}^{*}$ interaction are 216.7, -155.9 and $45.8 \mathrm{MeV}$.

\section{B. Describing the hot nucleus}

In the FTTF approach, the nucleon density profile for the hot nucleus is arrived at self-consistently. The details for obtaining the density in this method for the SkM* force are given in Ref. [15]; for the SBM interaction, they have also been presented earlier [14, 24]. However, for completeness, we present the salient features below.

The total energy of the nucleus is given by

$$
\begin{aligned}
E= & \int d \mathbf{r}\left[\varepsilon_{K}(r)+\varepsilon_{I}(r)+\varepsilon_{c}(r)\right] \\
& =E_{K}+E_{I}+E_{c}
\end{aligned}
$$

where $\varepsilon_{K}(r), \varepsilon_{I}(r)$ and $\varepsilon_{c}(r)$ are the kinetic, nuclear interaction and Coulomb interaction energy densities, respectively; $E_{K}, E_{I}$ and $E_{c}$ are the corresponding total energies. The energy densities are given by

$$
\varepsilon_{K}(r)=\frac{2}{h 3} \sum \int d \mathbf{p} \frac{p^{2}}{2_{m}} n_{\tau}(r, p, T)
$$




$$
\begin{aligned}
\varepsilon_{I}(r)= & \frac{2}{h^{3}} \sum_{\tau}\left[\frac { 1 } { h ^ { 3 } } \int \left\{v_{1}\left(\left|\mathbf{r}-\mathbf{r}^{\prime}\right|,\left|\mathbf{p}-\mathbf{p}^{\prime}\right|\right)\right.\right. \\
& \left.+v_{2}\left(\left|\mathbf{r}-\mathbf{r}^{\prime}\right|, \rho\right)\right\}\left\{C_{l} n_{\tau}\left(r^{\prime}, p^{\prime}\right)+C_{u} n_{-\tau}\left(r^{\prime}, p^{\prime}\right)\right\} \\
& \left.\times n_{\tau}(r, p)\right] d \mathbf{r}^{\prime} d \mathbf{p} d \mathbf{p}^{\prime} \\
\varepsilon_{c}(r)= & e^{2} \pi \rho_{p}(r) \int d r^{\prime}{r^{\prime}}^{2} \rho_{p}\left(r^{\prime}\right) g\left(r, r^{\prime}\right) \\
& \quad-\frac{3 e^{2}}{4 \pi}\left(3 \pi^{2}\right)^{1 / 3} \rho_{p}^{4 / 3}(r) .
\end{aligned}
$$

In these equations, $n_{\tau}(r, p, T)$ is the nucleon distribution function. In the Coulomb energy density, the first and second terms are the direct and exchange parts, respectively. Here, $\rho_{p}(r)$ is the proton density profile and

$$
g\left(r, r^{\prime}\right)=\frac{\left(r+r^{\prime}\right)-\left|r-r^{\prime}\right|}{r r^{\prime}} .
$$

In Eqs. (4) and (5), $\tau$ is the isospin index; if $\tau$ refers to a proton, $-\tau$ refers to a neutron and vice versa; the distribution function $n_{\tau}$ is determined from the minimization of the total thermodynamic potential $\Omega$ (= $\left.E-T S-\sum_{\tau} \mu_{\tau} N_{\tau}\right)$ [14]. Here $S$ is the total entropy of the system that can be obtained from Landau quasiparticle approximation as,

$$
S=-\frac{2}{h^{3}} \sum_{\tau} \int\left[n_{\tau} \ln n_{\tau}+\left(1-n_{\tau}\right) \ln \left(1-n_{\tau}\right)\right] d \mathbf{r} d \mathbf{p},(8)
$$

and $\mu_{\tau}$ are the nucleon chemical potentials. The distribution function is given as

$n_{\tau}(r, p, T)=\exp \left[\left(\frac{p^{2}}{2 m_{\tau}}+V_{\tau}^{0}+p^{2} V_{\tau}^{1}+V_{\tau}^{2}-\mu_{\tau}\right)\right]^{-1}$

In Eq. (9), $V_{\tau}^{0}(r)+p^{2} V_{\tau}^{1}(r)$ is the single-particle potential and $V_{\tau}^{2}(r)$ is the rearrangement potential originating from the density dependence of the interaction. The full expressions for the different components of the singleparticle potential and $V_{\tau}^{2}$ are given in Ref. [14] and are not repeated here. The effective $k$-mass of the nucleon $m_{\tau, k}(r)$ can be defined as

$$
m_{\tau, k}=\left[\frac{1}{m_{\tau}}+2 V_{\tau}^{1}\right]^{-1} .
$$

From Eq. (9), the nucleon density

$$
\rho_{\tau}(r)=\frac{2}{h^{3}} \int n_{\tau}(r, \mathbf{p}, T) d \mathbf{p}
$$

is obtained as, where

$$
A_{T}^{*}(r)=\frac{4 \pi}{h^{3}}\left[2 m_{\tau, k}(r) T\right]^{3 / 2},
$$

and $J_{k}(\eta)$ is the Fermi integral

$$
J_{k}(\eta)=\int_{0}^{\infty} \frac{x^{k}}{1+\exp (x-\eta)} d x
$$

with the fugacity $\eta$ given as

$$
\eta_{\tau}(r)=\left[\mu_{\tau}-\mathcal{V}_{\tau}(r)\right] / T .
$$

Here $\mathcal{V}_{\tau}(r)$ is the effective single-particle (SP) potential (Coulomb included), defined as

$$
\mathcal{V}_{\tau}(r)=\left(V_{\tau}^{0}(r)+V_{\tau}^{2}(r)+\delta_{\tau, p} V_{c}(r)\right) / T,
$$

$V_{c}(r)$ being the single-particle Coulomb potential,

$$
\begin{aligned}
V_{c}(r)= & 2 e^{2} \pi \int d r^{\prime} r^{\prime 2} \rho_{p}\left(r^{\prime}\right) g\left(r, r^{\prime}\right) \\
& -\frac{e^{2}}{\pi}\left(3 \pi^{2}\right)^{1 / 3} \rho_{p}(r)^{1 / 3} .
\end{aligned}
$$

From Eqs. (4) and (5), the kinetic and nuclear interaction energy densities can be simplified to

$$
\begin{aligned}
\varepsilon_{K}(r)= & \frac{2 \pi}{h^{3}} \sum_{\tau} \frac{1}{m_{\tau}}\left(2 m_{\tau, k} T\right)^{5 / 2} J_{3 / 2}\left(\eta_{\tau}(r)\right) \\
\varepsilon_{I}(r)= & \frac{1}{2} \sum_{\tau} \rho_{\tau}(r) V_{\tau}^{0}(r) \\
& +\frac{2 \pi}{h^{3}} \sum_{\tau}\left(2 m_{\tau, k} T\right)^{5 / 2} V_{\tau}^{1}(r) J_{3 / 2}\left(\eta_{\tau}(r)\right) .
\end{aligned}
$$

For a finite nucleus, the total energy density is the sum of Eqs. (6), (18) and (19). For infinite systems, the energy densities are space independent. For a particular density $\rho$, the kinetic and potential energies per nucleon can then be defined as $e_{K}=\varepsilon_{K} / \rho$ and $e_{I}=\varepsilon_{I} / \rho$, respectively.

At a large distance from the center, particularly for neutrons, $\mathcal{V}_{\tau} \sim$ is zero and the density becomes $\rho_{\tau} \sim$ $e^{\mu_{\tau} / T}$ which is zero at $T=0$ as $\mu_{\tau}$ is negative. At finite $T$, however, $\rho_{\tau}$ is not zero and takes a constant value with a nonzero pressure at the surface. This makes the system thermodynamically unstable. Moreover, the density profile becomes dependent on the size of the box in which calculations are done. This problem is overcome in the subtraction procedure 12, 25], where the hot nucleus, assumed to be a thermalized system in equilibrium with a surrounding gas simulating the effects of the evaporated nucleons, is separated from the embedding environment. This method is based on the existence of two solutions to the FTTF equations, one corresponding to the liquid phase with the surrounding gas $(l g)$ and the other cor- 
the hot nucleus in thermodynamic equilibrium is given by $\rho_{\tau, l}(r)=\rho_{\tau, l g}(r)-\rho_{\tau, g}(r)$ and it is independent of the choice of the box size. The conservation of nucleon number of each species $N_{\tau}$ of the hot nucleus gives

$$
\int\left[\rho_{\tau, l g}(r)-\rho_{\tau, g}(r)\right] d \mathbf{r}=N_{\tau}
$$

The energy $E$ of the nucleus is given by

$$
E=E_{l g}-E_{g},
$$

where $E_{l g}$ and $E_{g}$ are the total energies of the liquidgas system and the gas alone. From Eq. (8), the total entropy could be recast as [26],

$S=-\sum_{\tau} \int g_{\tau}\left(\varepsilon_{\tau}, T\right)\left[f_{\tau} \ln f_{\tau}+\left(1-f_{\tau}\right) \ln \left(1-f_{\tau}\right)\right] d \varepsilon_{\tau}$

where $f_{\tau}$ is the single-particle occupation function

$$
f_{\tau}\left(\varepsilon_{\tau}, \mu_{\tau}, T\right)=\left[1+\exp \left(\varepsilon_{\tau}-\mu_{\tau}\right) / T\right]^{-1},
$$

and $g_{\tau}$ is the subtracted single-particle level density. It is given as [24],

$$
\begin{aligned}
g_{\tau}\left(\varepsilon_{\tau}, T\right)= & \frac{4 \sqrt{2}}{\pi \hbar^{3}} \int\left[\left(m_{\tau, k}^{l g}\right)^{3 / 2} \sqrt{\varepsilon_{\tau}-\mathcal{V}_{\tau}^{l g}(r)}\right. \\
& \left.-\left(m_{\tau, k}^{g}\right)^{3 / 2} \sqrt{\varepsilon_{\tau}-\mathcal{V}_{\tau}^{g}(r)}\right] r^{2} d r
\end{aligned}
$$

where $\mathcal{V}_{\tau}^{i}$ is the single-particle potential, $i$ referring to $l g$ or $g$.

The free energy is calculated from $F=E-T S$. In terms of the occupation function, the density in the FTTF approximation could be written as

$$
\begin{aligned}
\rho_{\tau}^{i}(r)= & \frac{1}{2 \pi^{2} \hbar^{3}}\left(2 m_{\tau, k}^{i}\right)^{3 / 2} \int \sqrt{\varepsilon_{\tau}-\mathcal{V}_{\tau}^{i}} \\
& \times f\left(\varepsilon_{\tau}, \mu_{\tau}, T\right) d \varepsilon_{\tau} .
\end{aligned}
$$

\section{The energy-mass}

The effective mass $m^{*}$ has two components, the $k$-mass and the energy mass (or the $\omega$-mass); it is defined as

$$
m^{*}=m\left(\frac{m_{k}}{m}\right)\left(\frac{m_{\omega}}{m}\right),
$$

where $m$ is the nucleon mass.

The temperature-dependent $k$-mass is given by the momentum-dependent part of the single-particle potential as discussed earlier. The $\omega$-mass $m_{\omega}$ originates from the coupling of the single-particle motion with the collective degrees of freedom. The $m_{\omega}$ varies with position and temperature. A self-consistent calculation of the temperature-dependent $m_{\omega}$ is very involved and not within the scope of the present work. We have therefore taken a phenomenological form [17] for $m_{\omega}$ such that

$$
\underline{m_{\omega}}=1-0.4 A^{1 / 3} \exp \left[-\left(\frac{T}{-2}\right)^{2}\right] \frac{d \rho(r)}{2}
$$

The temperature $T$ and distance $r$ are measured in $\mathrm{MeV}$ fm units, $\rho(0)$ is the central density of the nucleon distribution in the nucleus. The collectivity refers to the liquid phase only. The density in the above equation is $\rho(r)=\rho_{l g}(r)-\rho_{g}(r) ; A$ refers to the liquid mass. This implies $m_{\omega}^{l g}=m_{\omega}$ and $m_{\omega}^{g}=m$.

To avoid the complexities arising from the selfconsistent calculation of the density profile with the inclusion of $\omega$-mass, we have adopted a realistic extension of the method given in Ref. [17]. This is described in some detail in Ref. [26].

The subtracted level density corresponding to Eq.(24) then modifies to

$$
\begin{aligned}
\tilde{g}_{\tau}\left(\varepsilon_{\tau}, T\right)= & \frac{4 \sqrt{2}}{\pi \hbar^{3}} \int\left[\left(m_{\tau, k}^{l g} \frac{m_{\omega}}{m}\right)^{3 / 2} \sqrt{\varepsilon_{\tau}-\mathcal{V}_{\tau}^{l g}(r) \frac{m}{m_{\omega}}}\right. \\
& \left.-\left(m_{\tau, k}^{g}\right)^{3 / 2} \sqrt{\varepsilon_{\tau}-\mathcal{V}_{\tau}^{g}(r)}\right] r^{2} d r
\end{aligned}
$$

the densities in the $l g$ or $g$ phase modify accordingly,

$$
\begin{aligned}
\tilde{\rho}_{\tau}^{i}(r)= & \frac{1}{2 \pi^{2} \hbar^{3}}\left[2 m_{\tau, k}^{i} \frac{m_{\omega}^{i}}{m}\right]^{3 / 2} \int \sqrt{\varepsilon_{\tau}-\mathcal{V}_{\tau}^{i} \frac{m}{m_{\omega}^{i}}} \\
& \times f\left(\varepsilon_{\tau}, \tilde{\mu}_{\tau}, T\right) d \varepsilon_{\tau} .
\end{aligned}
$$

The chemical potential $\mu_{\tau}$ has modified to $\tilde{\mu}_{\tau}$ to conserve the particle numbers in the nucleus

$$
N_{\tau}=\int \tilde{g}_{\tau}\left(\varepsilon_{\tau}, T\right) f_{\tau}\left(\varepsilon_{\tau}, \tilde{\mu}_{\tau}, T\right) d \varepsilon_{\tau} .
$$

\section{The symmetry coefficients}

For nuclear matter, the symmetry coefficient at density $\rho$ can be defined as,

$$
e_{\text {sym }}(T)=[e(\rho, X, T)-e(\rho, X=0, T)] / X^{2},
$$

where $e$ is the energy per nucleon and $X$ is the asymmetry parameter $\left.X=\left(\rho_{n}-\rho_{p}\right) / \rho\right)$. Here $\rho_{n}$ and $\rho_{p}$ are the neutron and proton densities $\left(\rho=\rho_{n}+\rho_{p}\right)$. The kinetic and potential parts of the symmetry coefficients $e_{\text {sym }}(k)$ and $e_{\text {sym }}(v)$ can likewise be defined replacing $e$ in the square brackets in Eq. (31) by $e_{K}$ and $e_{I}$, respectively.

For a nucleus of mass $A$, the symmetry coefficient, in the spirit of the liquid-drop model, can also be defined as

$$
e_{\text {sym }}(T)=[e(N, Z, T)-e(A / 2, A / 2, T)] / X^{2} .
$$

Here the asymmetry parameter is $X=(N-Z) / A$. In this equation, $e$ is the energy per particle of the nucleus barring the Coulomb contribution. For relatively heavy nuclei, stable systems are usually isospin-asymmetric, there the definition given by Eq.(32) may not be operative. In such systems, $e_{\text {sym }}$ could be calculated from 
where $X_{1}$ and $X_{2}$ are the asymmetry parameters of the nuclear pair. Similar definitions follow for symmetry free energy coefficient $f_{\text {sym }}$ on replacing energy $e$ by the free energy per particle $f$.

The value of the symmetry coefficient obtained from Eqs. (32) and (33) depends on the choice of the nuclear pair, its value is therefore not unambiguous for a particular nucleus. However, the local density approximation (LDA) could be used to define the symmetry coefficient for a specific nucleus once its density profile is known. In the LDA, it is given as [27]

$$
\begin{aligned}
e_{\text {sym }}(T)\left(\frac{N-Z}{A}\right)^{2}= & \frac{1}{A} \int \rho(r) e_{\text {sym }}^{\infty}[\rho(r), T] \\
& \times\left[\frac{\rho_{n}(r)-\rho_{p}(r)}{\rho(r)}\right]^{2} d \mathbf{r} .
\end{aligned}
$$

Here $e_{s y m}^{\infty}[\rho(r), T]$ is the symmetry energy coefficient of infinite matter at temperature $T$ at a value of the density $\rho(r)$. The local isospin density is given by $\left[\rho_{n}(r)-\rho_{p}(r)\right]$.

\section{RESULTS AND DISCUSSIONS}

As already stated, for the microscopic finite temperature Thomas-Fermi calculations, we have chosen the $\mathrm{SkM}^{*}$ and the SBM effective interactions. Both these interactions reproduce the ground state properties of atomic nuclei and also of nuclear matter fairly well. For normal nuclear matter at $T=0$, these interactions have symmetry coefficient $e_{\text {sym }} \sim 31.0 \mathrm{MeV}$, a commonly accepted value with some support from experimental analyses [3]. Below the saturation density, both these interactions display nearly the same density dependence for $e_{s y m}$ as shown in Fig. 1. The symmetry coefficient obtained from the SBM interaction (given by the red line) looks a little stiffer beyond the saturation density. It would be interesting to compare the symmetry coefficients of cold nuclei obtained from these interactions with phenomenological values. This is shown in panels (a) and (b) of Fig. 2; the full green lines in both the panels correspond to the upper and lower bounds for $e_{\text {sym }}$ as given in Ref. [2] as

$$
e_{\text {sym }}=\frac{\alpha}{1+\frac{\alpha}{\beta} A^{-1 / 3}},
$$

where $\alpha=31.0 \mathrm{MeV}$ and $\frac{\alpha}{\beta}=2.4 \pm 0.4$. The black circles and red triangles refer to calculations with $\mathrm{SkM}^{*}$ and SBM interactions, respectively. The upper panel corresponds to calculations done in the difference method using Eq. (33). The nuclear pairs $\left(A, Z_{1}\right)$ and $\left(A, Z_{2}\right)$ collectively written as $\left(A, Z_{1}, Z_{2}\right)$ are chosen as $(26,10,12),(40,16,18),(56,24,26),(80,34,36)$, $(120,50,52),(150,60,62), \quad(197,77,79)$, and $\quad(238,90,92)$. These results are representative, the values of $e_{s y m}$ depend on the choice of nuclear pairs as shown later. The lower panel displays results calculated in the LDA

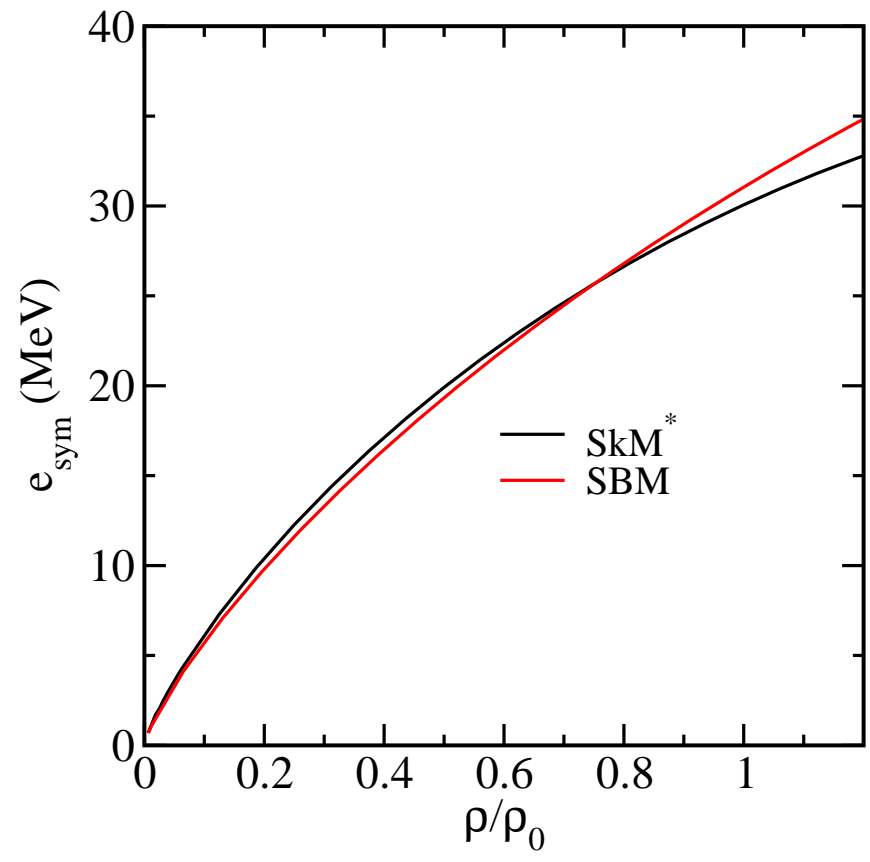

FIG. 1: (color online)The symmetry energy coefficient $e_{\text {sym }}$ for nuclear matter at $T=0$ as a function of density with $\mathrm{SkM}^{*}$ (black) and SBM (red) interactions.

the $\beta$-stability line; they are $(26,12),(40,18),(56,26)$, $(80,34),(120,50),(150,62),(197,79)$, and $(238,92)$, respectively. In the LDA, the values of $e_{s y m}$ are seen to have a relatively weak dependence on the choice of the asymmetry parameter $X$. Compared to those in SBM, the $\mathrm{SkM}^{*}$ values are somewhat lower. The values of $e_{\text {sym }}$, however, are seen to lie within the phenomenological limits, particularly in the LDA. The difference in results in $e_{\text {sym }}$ for finite nuclei in spite of the apparent similarity in its density dependence for infinite nuclear matter stems from the fact that the SBM is a finite range interaction as opposed to $\mathrm{SkM}^{*}$ (zero range) which results in somewhat different density profiles of nuclei.

Before embarking on the evaluation of the temperature dependence of the symmetry coefficients for finite systems, it may be worthwhile to see how $e_{\text {sym }}$ or $f_{\text {sym }}$ behave with increasing temperature for infinite nuclear matter. At saturation density, we find that both $e_{\text {sym }}$ and $f_{\text {sym }}$, in the temperature range we have studied, have a very weak temperature dependence with both the interactions chosen. At relatively low densities, $e_{s y m}$ is seen to decrease with temperature, in consonance with that reported earlier by Xu et.al., 6] and Moustakidis [28]. At these densities, $f_{\text {sym }}$, however, displays a comparably more prominent rise, in fair agreement with those obtained earlier [6, 27]. This is displayed in Fig. 3 at $\rho=\rho_{0} / 8\left(\rho_{0}=\right.$ saturation density $)$ for the SBM interaction. Results with $\mathrm{SkM}^{*}$ interaction do not show any different behavior and are therefore not shown. The fall 


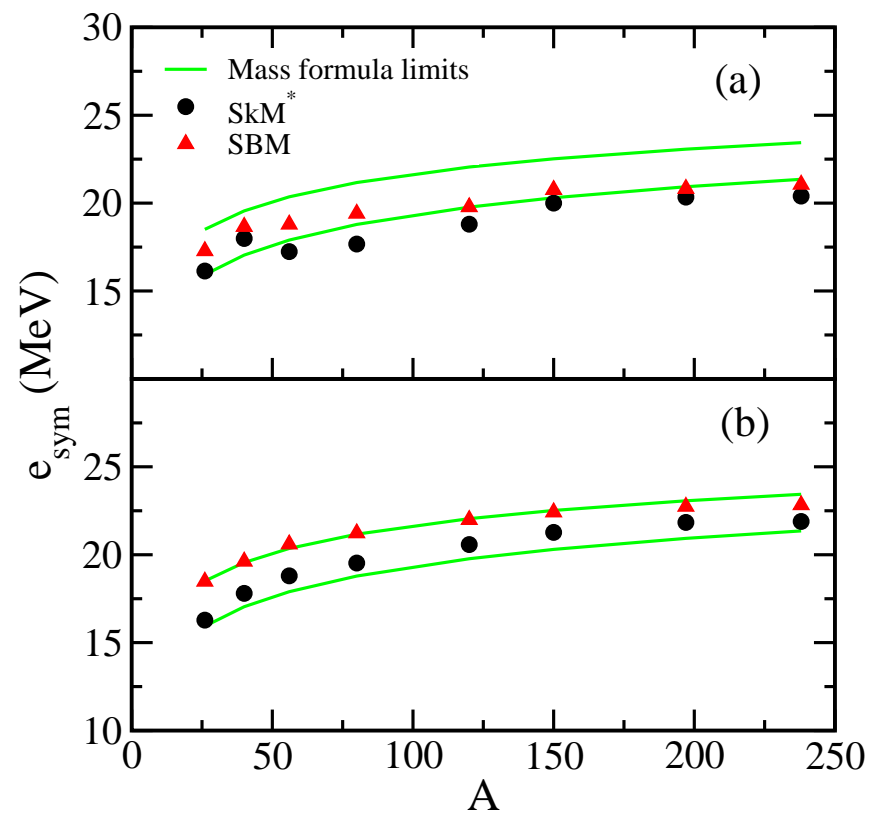

FIG. 2: (color online)The dependence of symmetry energy coefficient $e_{\text {sym }}$ on nuclear mass number with $\mathrm{SkM}^{*}$ (black circles) and SBM (red triangles) interactions. The limits in the nuclear mass formula as given in Ref. [2] are also shown (green lines). The upper and lower panels correspond to calculations using Eqs. (33) and (34), respectively. For details, see text.

low density is essentially due to the decrement in its kinetic energy part $e_{\text {sym }}(k)$ (shown by the green line). This part of the symmetry energy decreases at lower densities as Pauli-blocking becomes less important because of increased diffuseness of nucleon Fermi surfaces with temperature. The potential component $e_{\text {sym }}(v)$ (black line) decreases more slowly. The increase of $f_{\text {sym }}$ with temperature can be understood from the fact that

$$
f_{\text {sym }}=e_{\text {sym }}-T \frac{s_{\text {sym }}}{X^{2}}
$$

where $s_{\text {sym }}$, the symmetry entropy is negative and is $\sim-\frac{1}{2} X^{2}$ [29] for dilute nuclear matter. Thus even if $e_{\text {sym }}$ falls slower, the increase in $f_{\text {sym }}$ (lower panel) is noticeable.

In Fig. 4, the temperature dependence of the symmetry energy coefficients and their kinetic and potential components for a nucleus $A=56$ is shown for the SBM interaction. The upper panel represents calculations in the difference method where Eq. (33) is exploited to obtain the symmetry coefficients with $Z_{1}=24$ and $Z_{2}=26$. The density profiles of the nuclei have been calculated with Coulomb switched on, however, the symmetry coefficients are calculated from the energies obtained after subtraction of the Coulomb contribution. The lower panel shows the calculations in LDA with the use of Eq. (34) for the nuclei with $Z=24$ and 26 . The difference method shows that for nuclei, the main contribution to

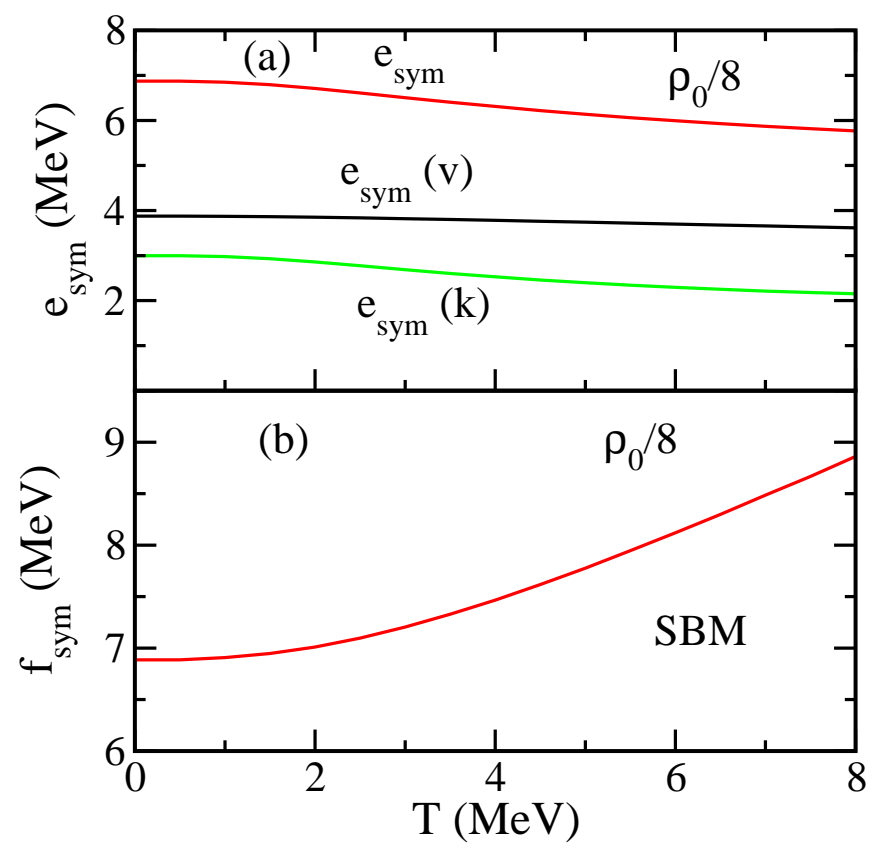

FIG. 3: (color online) In the upper panel the temperature evolution of kinetic (green) and potential (black) components $e_{\text {sym }}(k)$ and $e_{\text {sym }}(v)$, respectively, of symmetry energy coefficient along with their sum $e_{\text {sym }}$ (red) are shown for nuclear matter at a density $\rho_{0} / 8$ calculated with SBM interaction. In the lower panel, the same is shown for the total symmetry free energy coefficient (red) $f_{\text {sym }}$.

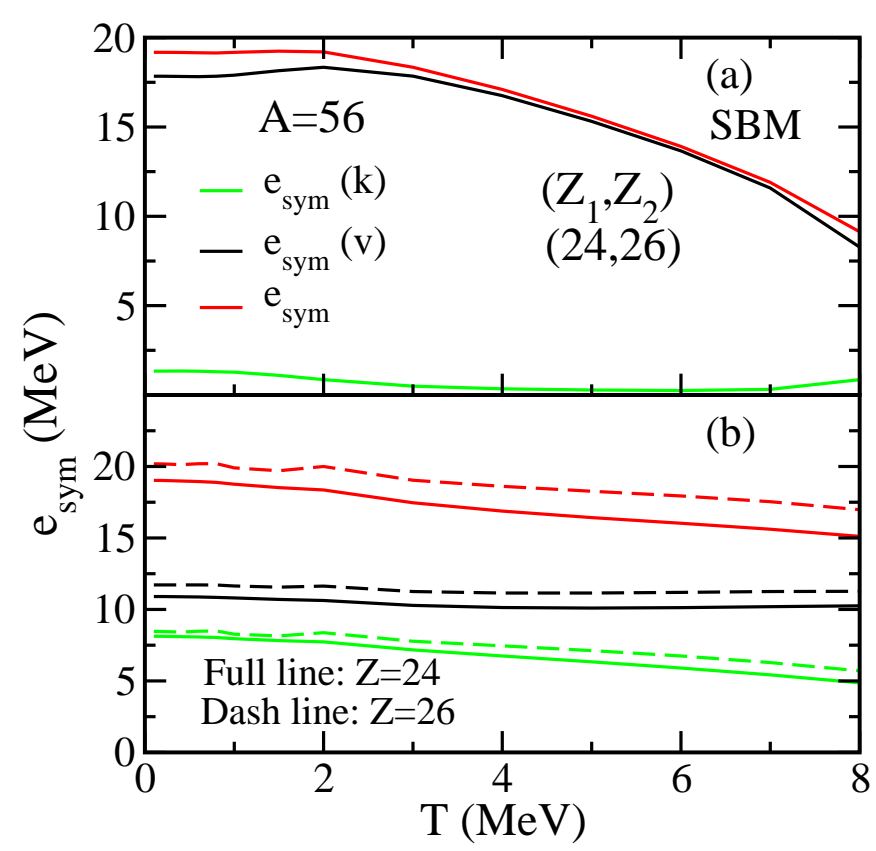

FIG. 4: (color online) The upper panel represents the temperature evolution of kinetic (green) and potential (black) components of symmetry energy coefficient along with their sum $e_{\text {sym }}$ (red) obtained from $\mathrm{A}=56$ isobaric pair with $\mathrm{Z}=24$ and 26 using SBM interaction. In the lower panel, the same is shown for these isobaric nuclei individually in the local density approximation. 


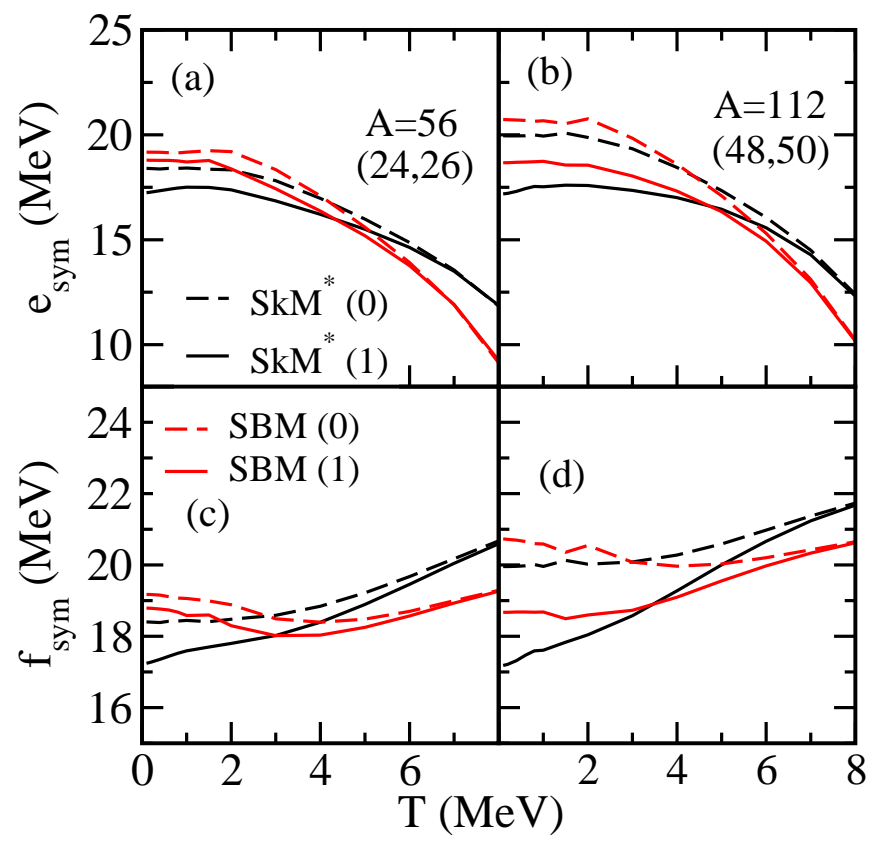

FIG. 5: (color online) The temperature dependence of $e_{\text {sym }}$ using $\mathrm{SkM}^{*}$ interaction with $\left(\mathrm{SkM}^{*}(1)\right)$ and without $\left(\mathrm{SkM}^{*}(0)\right)$ inclusion of $\omega$-mass are shown in panel (a) by black full and dash lines, respectively, for $\mathrm{A}=56$ calculated with $\mathrm{Z}=(24,26)$ isobaric pair. The red lines represent the same for SBM interaction. The same is shown in panel (b) for $A=112$ using $\mathrm{Z}=(48,50)$ isobaric pair. The panels (c) and (d) represent the same for $f_{\text {sym }}$.

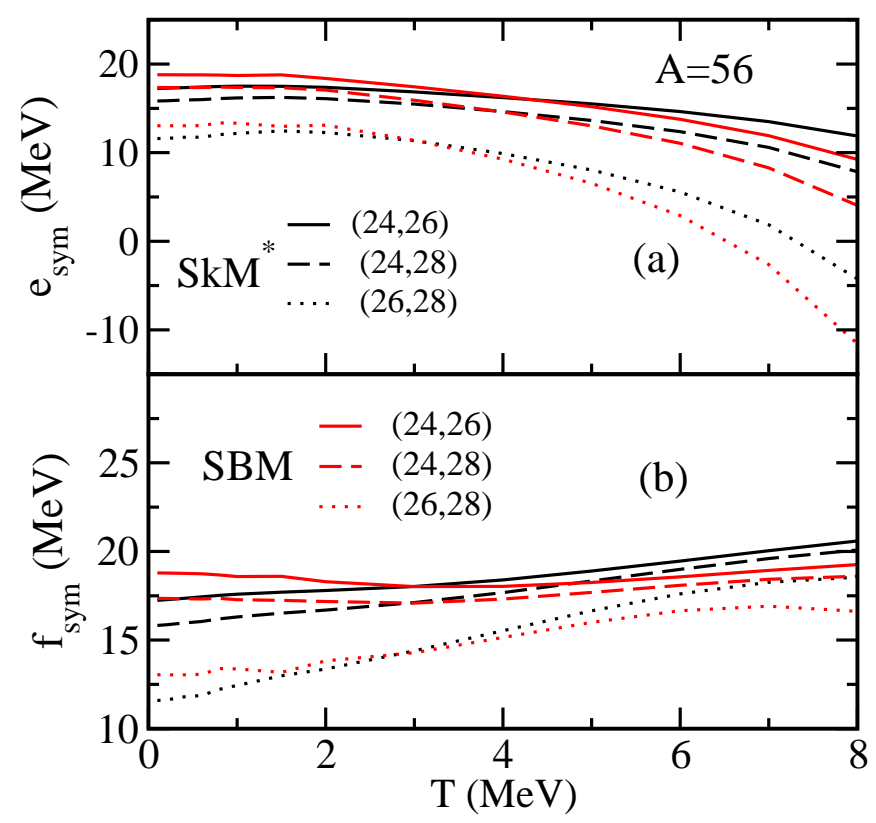

FIG. 6: (color online) The temperature dependence of $e_{\text {sym }}$ (upper panel) and $f_{\text {sym }}$ (lower panel) for the nucleus $\mathrm{A}=56$. The black and red lines correspond to results with SkM* and SBM interaction, respectively. The numbers in the parentheses refer to charge numbers of the different isobaric pairs used to calculate the symmetry energies.

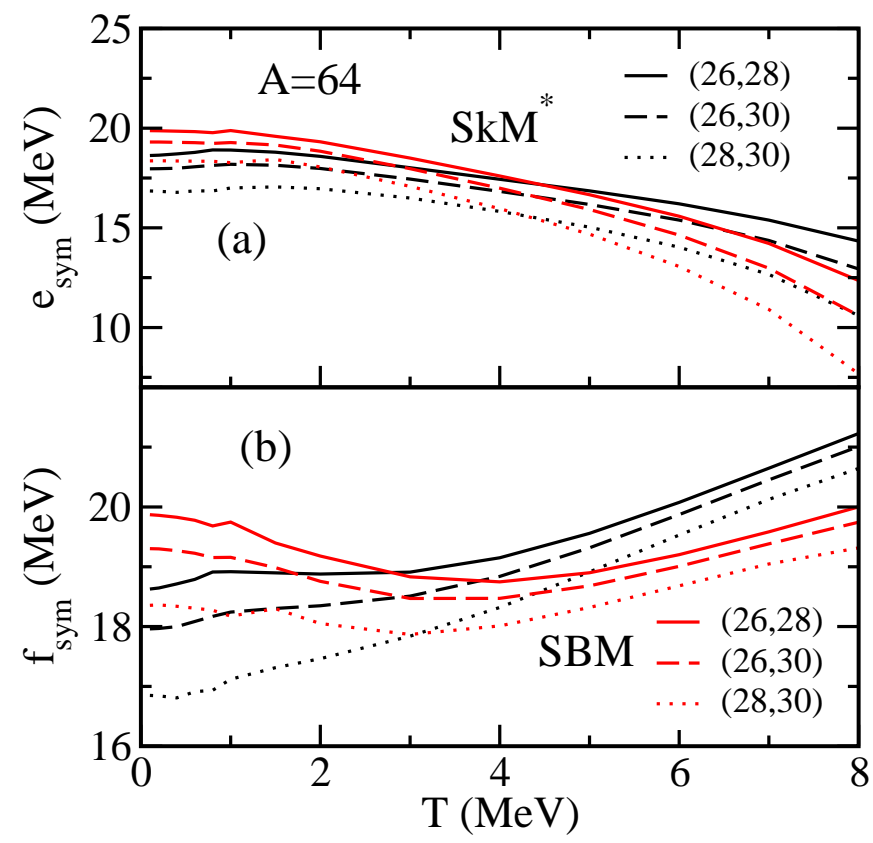

FIG. 7: (color online) The same as in Fig. 6 for the nucleus $\mathrm{A}=64$ with isobar pairs as shown in the parentheses.

part (the black line), $e_{\text {sym }}(k)$ (the green line) is quite small. The symmetry coefficient is nearly constant upto $\sim 2 \mathrm{MeV}$, but then falls substantially with temperature. The calculations in the LDA, for the isobars with $Z=24$ and 26 , however, show a very weak temperature dependence for the symmetry coefficients, which comes mostly from the surface region; the temperature dependence of infinite matter is reflected in these calculations for finite nuclei. Here the contributions from $e_{s y m}(v)$ and $e_{s y m}(k)$ are comparable.

Till now, calculations have been reported without the inclusion of the effects of the $\omega$-mass. As can be seen from Eq. (27), $m_{\omega} / m$ may be significantly larger than unity, more so for heavier nuclei and at lower temperatures. This would decrease the effective kinetic energy of the system and may also decrease the corresponding symmetry energy. The effects of $\omega$-mass on the symmetry coefficients $e_{\text {sym }}$ and $f_{\text {sym }}$ are shown for both the SBM (red lines) and SkM* (black lines) interactions in Fig. 5. The number (1) in parentheses refers to calculations (full lines) with inclusion of the effects of $\omega$-mass, (0) refers to calculations (dashed lines) without it. The results from the difference method are only reported. A relatively light $(A=56)$ and a medium-heavy $(A=112)$ nucleus are chosen for this purpose. As seen from the upper and lower panels in the figure, $e_{\text {sym }}$ and $f_{\text {sym }}$ decrease with the inclusion of $\omega$-mass effects. The dilution of the effects due to energy mass with increase in temperature is evident from the merging of the full and dashed lines with the same color representing a given interaction at high $T$. As stated earlier, the reduction in the symmetry coefficients with inclusion of $\omega$-mass is more prominent 


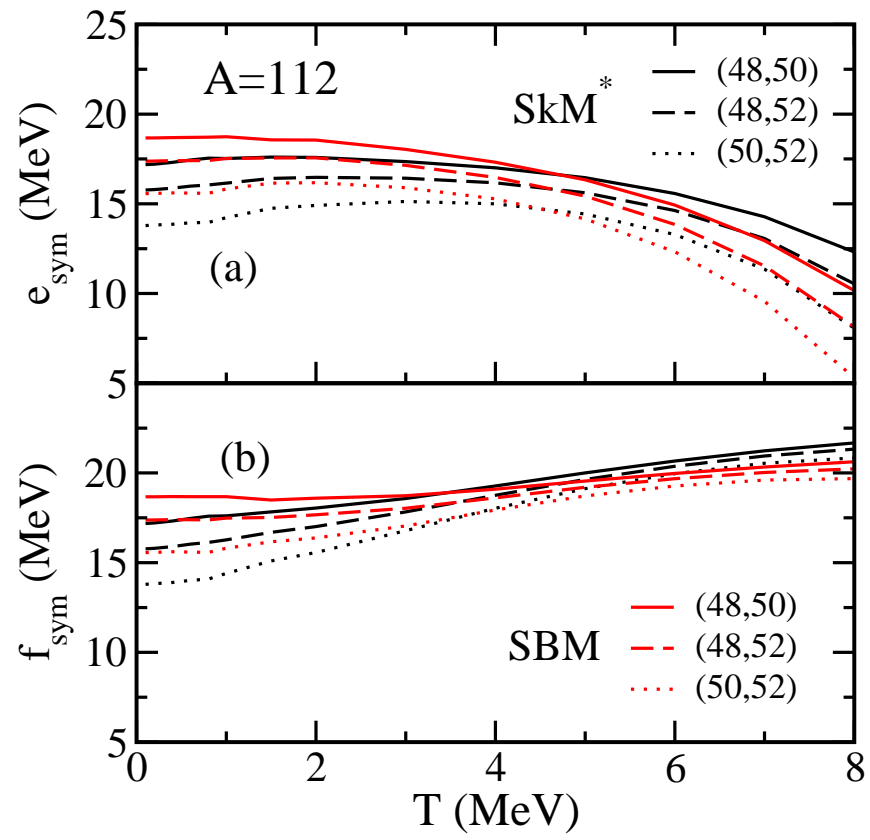

FIG. 8: (color online) The same as in Fig. 6 for the nucleus $\mathrm{A}=112$.

The dependence of the nuclear symmetry energies on the choice of nuclear pairs for a given isobar is shown in Fig. 6 for $A=56$. The chosen nuclear pairs $\left(Z_{1}, Z_{2}\right)$ are $(24,26),(24,28)$, and $(26,28)$. The upper panel displays the temperature dependence of $e_{\text {sym }}$ and the lower panel shows the same for $f_{\text {sym }}$. The figure shows the sensitivity of the symmetry coefficients on the choice of the nuclear pair; however, for all the cases, $e_{\text {sym }}$ decreases whereas $f_{\text {sym }}$ increases with temperature. With the nuclear pair $Z_{1}=26$ and $Z_{2}=28, e_{\text {sym }}$ even becomes negative at high temperature. This violates the general understanding of the symmetry energy; this negativity is likely to arise from the Coulomb polarization of the density profiles of nuclei. We have checked that calculations with Coulomb switched off never give negative symmetry energy coefficients. The different interactions SkM* and SBM yield somewhat different values for $e_{\text {sym }}$ and $f_{s y m}$; in general, values obtained for the symmetry coefficients with the $\mathrm{SkM}^{*}$ interaction are lower compared to those for SBM interaction at lower temperatures. A crossover, however, is generally seen at higher temperatures. Calculations have been performed for nuclear isobars with $A=64$ and $A=112$ also. They are reported in Fig. 7 and Fig. 8. These results follow nearly the same trend as those for $A=56$.

\section{CONCLUDING REMARKS}

We have performed calculations for the temperature dependence of the symmetry energy and symmetry free energy coefficients of atomic nuclei in a Thomas-Fermi model. Calculations have been done with two effective interactions, namely, the SkM* and the SBM. The subtraction procedure has been employed for modeling the hot nucleus. Effects of the coupling of the surface phonons to the intrinsic particle motion have been taken into account through a phenomenological parametric form of the nucleon $\omega$-mass. The $\omega$-mass increases the nucleon effective mass at relatively low temperatures, this has an appreciable effect on the symmetry coefficients which decrease noticeably once effects due to $m_{\omega}$ are taken into account.

Questions may arise about the proper definition of the symmetry coefficients for finite nuclei. We have taken two definitions, one derived from the usual difference method [11], another from the local density approximation (LDA). In both approaches, we find that $e_{\text {sym }}$ decreases with temperature, $f_{\text {sym }}$ shows the opposite trend. In the LDA, the fall in $e_{\text {sym }}$ is slow, in the difference method, this fall with temperature is much stronger. In this method, the results, however, depend sensitively on the choice of the nuclear pairs taken to evaluate the symmetry energies. Occasionally, the symmetry coefficients may even be negative, this is violative of the general concept of the symmetry coefficients.

The ambiguities arising from the different definitions for symmetry coefficients of finite nuclear systems may possibly be overcome by calculating the ground state energies of a host of nuclei over the periodic table with a suitable choice of interaction in a microscopic framework, such as Hartree-Fock ( $\mathrm{HF}$ ) and then finding the mass parameters $a_{v}, a_{s}$ etc. in the spirit of the liquiddrop model. The calculations can be extended to various temperatures employing finite temperature HF method and then find the temperature dependence of the mass parameters which is likely to give ambiguity-free temperature dependence of the symmetry coefficients. This is, however, an ambitious program and the work is in progress.

\section{Acknowledgments}

J.N.D and S.K.S acknowledge support of DST, Government of India.
[1] W. D. Myers and W. J. Swiatecki, Nucl. Phys. A601, 141 (1996).

[2] P.Danielewicz, Nucl. Phys. A727, 233 (2003).

[3] D. V. Shetty, S. J. Yennello, and G. A. Souliotis, Phys.
Rev. C 75, 034602 (2007).

[4] A. W. Steiner, M. Prakash, J. M. Lattimer, and P. J. Ellis, Phys. Rep. 411, 325 (2005).

[5] H.-Th. Janka, K. Langanke, A. Marek, G. Martínez- 
Pinedo, and B. Müller, Phys. Rep. 442, 38 (2007).

[6] Jun Xu, Lie-Wen Chen, Bao-An Li, and Hong-Ru Ma, Phys. Rev. C 75, 014607 (2007).

[7] C. J. Horowitz and A. Schwenk, Nucl. Phys. A 776, 55 (2006).

[8] S. Mallik, J. N. De, S. K. Samaddar, and Sourav Sarkar, Phys. Rev. C 77, 032201 (R) (2008).

[9] P. Donati, P. M. Poacher, P. F. Bortignon, and R. A. Broglia, Phys. Rev. Lett. 72, 2835 (1994).

[10] R. W. Hasse and P. Schuck, Nucl. Phys. A 445, 205 (1985).

[11] D. J. Dean, K. Langanke, and J. M. Sampaio, Phys. Rev. C 66, 045802 (2002).

[12] E. Suraud, Nucl. Phys. A 462, 109 (1987).

[13] D. Bandyopadhyay, C. Samanta, S. K. Samaddar, and J. N. De, Nucl. Phys. A 511, 1 (1990).

[14] J. N. De, N. Rudra, Subrata Pal, and S. K. Samaddar, Phys. Rev. C 53, 780 (1996).

[15] M. Brack, C. Guet, and H. B. Hakansson, Phys. Rep. 123, 275 (1985).

[16] M. Prakash, J. Wambach, and Z. Y. Ma, Phys. Lett. B 128, 141 (1983).

[17] S. Shlomo and J. B. Natowitz, Phys. Lett. B 252, 187 (1990).
[18] J. N. De, S. K. Samaddar, X. Vinas, and M. Centelles, Phys. Lett. B 638, 160 (2006).

[19] R. G. Seyler and C. H. Blanchard, Phys. Rev. C 124, 227 (1961).

[20] W. D. Myers and W. J. Swiatecki, Ann. Phys. (N. Y.) 55, 395 (1969).

[21] W. D. Myers and W. J. Swiatecki, Ann. Phys. (N. Y.) 204, 401 (1990).

[22] M. M. Majumdar, S. K. Samaddar, N. Rudra, and J. N. De, Phys. Rev. C 49, 541 (1994).

[23] N. Rudra and J. N. De, Nucl. Phys. A 545, 608 (1992).

[24] S. K. Samaddar, J. N. De, X. Viñas, and M. Centelles, Phys. Rev. C 75, 054608 (2007).

[25] P. Bonche, S. Levit, and D. Vautherin, Nucl. Phys. A 436, 265 (1985).

[26] J. N. De, S. Shlomo, and S. K. Samaddar, Phys. Rev. C 57, 1398 (1998).

[27] S. K. Samaddar, J. N. De, and X. Viñas, and M. Centelles, Phys. Rev. C 76, 041602 (R) (2007).

[28] Ch. C. Moustakidis, Phys. Rev. C 76, 025805 (2007).

[29] J. N. De and S. K. Samaddar, Phys. Rev. C 78, 065204 (2008). 